II.

Aus der medizinischen Klinik des städtischen Krankenhanses zu Frankfurt a. M.

Direktor Professol Dr. Schwenkenbecher.

Über die Wirkung der die Körpertemperatur beeinflussenden Gifte auf Tiere ohne Wärmeregulation.

I. Nitteilung: Natrium salicylicnm, Antipyrin, Chinin, Morphin.

Von

R. Isenschmid.

(Mit 4 Kurven.)

Neuere Arbeiten aus der Heidelberger medizinischen Klinik haben uns gelehrt, durch Ausschaltung des Einflusses der vegetativen Zentren an der Basis des Zwischenhirns auf den Organismus die Wärmeregulation! ganz oder partiell auszuschalten. Am leichtesten gelingt diese Ausschaltung durch einen Eingriff an Ort und Stelle im Gehirn ${ }^{1}$ ) oder durch quere Durchtrennung des Rückenmarkes oberhalb des ersten Dorsalsegmentes. ${ }^{2}$ ) Durchschneidet man das Rückenmark weiter unten, schaltet man nur den physikalischen Anteil des Wärmeregulationsmechanismus aus. ${ }^{2},{ }^{3}$ ) Wird dagegen anßerdem noch der Nervus sympathicus doppelseitig durchtrennt, ist auch die chemische Wärmeregulation aufgehoben. Einen ähnlichen Einfluß hat die Kombination der Brustmarkdurchschneidung mit der Durchtrennung der Vagi unter dem Zwerchfell. ${ }^{4}$ )

1) Isenschmid und Krehl, Über den Einfluß des Gehirns anf die Wärmeregulation. Arch. f. exper. Path. u. Pharmakol. Bd. 70, 1912.

2) Freund und Strasmann, Zur Kenntnis des nervösen Mechanismus der Wärmeregulation. Ebenda Bd. 69, 1912.

3) Freund und Grafe, Untersuchungen über den nervösen Mechanismus der Wärmeregulation. Ebenda Bd. 70, 1912.

4) Freund, Die Bedeutung der Vagi fir die Wärmeregulation. Arch. $f$. exper. Path. п. Pharmakol. Bd. 72, 1913 
$O b$ es vielleicht gelingt, durch Unterbrechung des Sympathikus und Vagus allein, oder durch einen sonstigen Eingriff die chemische Wärmeregulation allein aufzuheben ohne Störung des physikalischen Regulationsvermögens, ist zurzeit noch nicht nachgewiesen. Für den Stoffwechselversuch könnte ein solches Verfahren von größter Bedeutung sein.

Rubner1) hat zuerst gezeigt, daß manche den Gesamtstoffwechsel in seiner Intensität verändernden Einflüsse durch die chemische Wärmeregulation verdeckt, ganz oder teilweise kompensiert werden können.

Die »spezifisch dynamische Wirkung" der Nahrungsstoffe trat nur dann voll und ganz in Erscheinung, wenn die Versuchstiere bei einer hohen Außentemperatur gehalten wurden, bei einer Temperatur, bei welcher die Verbrennungen im Körper im nuichternen Zustande ein Minimum erreichen. Wurde bei niedrigeren Temperaturen experimentiert, so deckten die durch die Nahrungszufuhr bedingten Verbrennungen teilweise den ohnehin stattfindenden intensiveren Stoffumsatz, so daß die Steigerung der Verbrennungen gegenüber dem Hungerzustande dann geringer ausfiel als bei höherer Lufttemperatur.

Rubner hat also zuerst gezeigt, daß die chemische Wärmeregulation bei Untersuchungen des Gesamtstoffwechsels ausgeschaltet werden müßte, und er hat dies anch planmäßig - eben durch Versetzen in eine hochtemperierte Umgebung - in vielen Versuchen nach Möglichkeit getan.

Ob die ehemische Wärmeregulation bei hohen Außentemperaturen unter allen Versuchsbedingungen ausgeschaltet bleibt, ist zum mindesten zweifelhaft. Für das Studium der den Gesamtstoffwechsel herabsetzenden Einflusse kann eine solche Versuchsanordnung jedenfalls nicht genügen, denn es ist wahrscheinlich, daß auch bei hoher Lufttemperatur bei einem Tier mit intaktem Nervensystem die chemische Wärmeregulation mit ihrem steigernden Einflusse wieder einsetzen wird, wenn ein den Gesamtstoffwechsel herabsetzender Einfluß in Wirksamkeit tritt. Hier kann nur die operative Ausschaltung der chemischen Wärmeregulation eine Kompensation verhindern und der durch das Agens hervorgerufenen Herabsetzung des Stoffwechsels zu ihrer vollen Entfaltung: verhelfen.

Demnach miißten eigentlich alle den Gesamtstoffwechsel herab-

1) Die Gesetze des Energieverbrauches bei der Ernährung. Leipzig und Wien 1902; dort sind anch die älteren Arbeiten über dieses Thema angeführt. 
setzenden oder steigernden Einflüsse an Tieren, nach operativer Ausschaltung der chemischen Wärmeregulation, von neuem untersucht werden.

Vor allem kann nun endlich der vom zentralen Regulationsmechanismus unabhängige Teil des Einflusses der Fiebererreger auf den Stoffwechsel rein studiert werden. Ich beabsichtige solche Versuche, deren Vorarbeiten mich seit mehr als einem Jahre beschäftigen, in größerem Umfange durchzuführen. Anch von anderer Seite, speziell von Leschke1), sind uns nenerdings solche Versuche in Aussicht gestellt. Es ist zu hoffeu, daß diese Fragen von vielen Seiten in Angriff genommen werden, denn durch einige wenige Untersuchnngen wird dieses Kapitel auch jetzt kaum endgültig and erschöpfend geklärt werden. Auch der Einfluß der Nahrungsaufnahme anf die Gesamtsumme der Verbrennungen könnte nun mit Aussicht auf neue Ergebnisse von neuem studiert werden, ebenso viele andere Einflisse, z. B. der der Maskelarbeit oder der Ausschaltung 'maneher Organfunktionen und besonders der pharmakologischen Agentien auf den Gesamtstoffwechsel, denn wir wissen nicht, wieviel von diesen Einflüssen bei den bisher möglichen Versuchsanordnungen durch die ehemische Wärmeregulation verdeckt worden ist. Vielleicht sind auch umgekehrt manche Einflisse, besonders von Arzneimitteln, welche bisher durch Beeinflussung der Wärmeregulationszentren erklärt worden sind, noch nach deren Ausschaltung vorhanden und werden nun in ein anderes Licht gerïckt werden können.

Alle diese Versuche müßten nach Ausschaltung der zentralen Wärmeregulation mit den exaktesten Methoden ansgeführt werden, am besten mit direkter Kalorimetrie, mindestens aber mit Gaswechselbestimmungen bei kurz dauernden Veränderungen, mit Kohlenstoffund Stickstoff-Bilanzen, bei in längeren Perioden sich abspielenden Prozessen. Solche methodisch komplizierte Untersuchungen habe ich aus Mangel an Zeit und an Hilfsmitteln bisher nicht ansfubren können, sondern einen anderen Weg beschritten, der mir geeignet schien, die gleichen Resultate, wenn anch in weniger exakter Weise, auf einfachere Art zu erreichen.

Ich habe Tiere, deren Wärmeregulation ausgeschaltet war, im Hungerzustande in einem sehr gleichmäßig temperierten Wärmeschrank bei einer Temperatur gehalten, bei welcher ibre Körpertemperatur normale Höbe hat und habe jede auf einen toxischen

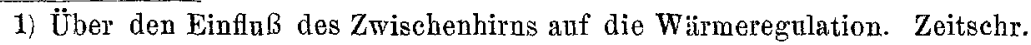
f. exper. Path. u. Ther p. Bd. 14, 1913. 
oder anderen Einfluß stattfindende Schwankung der Körpertemperatur als Ausdruck gesteigerter oder verminderter Wärmebildung betrachtet.

Wenn dieVeränderungen der Körpertemperatur eines solchen Tieres als genaues Abbild der Schwankungen der Größe der gesamten Verbrennungen angesehen werden dürfen, müssen folgende Voranssetzungen erfüllt sein.

Erstens müssen die Wärmebildung und die Wärmeabgabe im unbeeinflußten Zustande absolut gleichmäßig verlaufen, mindestens während einer, entsprechend der Daner unserer Versuche, auf mehrere Stunden sich bemessenden Zeitdauer.

Zweitens dürfen die zu prüfenden Agentien nur die Wärmebildung, nicht aber die Wärmeabgabe beeinflussen.

Das Verhalten der Versuchstiere spricht dafür, daß dies in der Tat im ganzen zutrifft. Der exakte, auf kalorimetrischem Wege zu fübrende Beweis dafür steht allerdings noch aus. Wir glauben aber zeigen zu können, daB wir trotzdem die genannten Voraussetzungen bis zu einem gewissen Grade als erfüllt betrachten dürfen.

Tiere, deren physikalische und chemische Wärmeregulation operativ ausgeschaltet ist, haben, wie schon früher gezeigt wurde, im Hunger nur bei einer ganz bestimmten, von Tier zu Tier verschiedenen Lufttemperatur eine normale und konstante Körpertemperatur. Bleibt die Außentemperatur und die Ventilation des Wärmeschrankes ganz konstant, ist die Konstanz der Körpertemperator des operierten Tieres eine sehr große. Es gelingt in guten Thermostaten die Körpertemperatur eines solchen Kaninchens halbe, ja ganze Tage lang Stunde für Stunde so konstant zu erhalten, daß keine Schwankungen von mehr als zwei Zehntel Graden vorkommen. Kaninchen mit intakter Wärmeregulation haben auch bei konstantester Außentemperatur und im Hungerzustande niemals - ich verfüge uiber sehr zahlreiche, stindliche Messungen an normalen Tieren - eine auch nur annähernd so geradlinig verlaufende Tageskurve.

Dieser absolut geradlinige, horizontale Verlauf der Temperaturkurve unserer Tiere ohne Wärmeregulation ist nur möglich, wenn die Wärmebildung und die Wärmeabgabe sehr gleichmäßig verlaufen.

Wird die Wärmeabgabe durch Veränderungen der Außentemperatur verändert, ändert sich sofort die Körpertemperatur, und zwar fällt die Körpertemperatur z. B. bei einer Herabsetzung der Außentemperatur um einige Grade ganz gleichmäßig, ohne die geringsten Unebenheiten der Kurve ab. Die Verbrennungen müssen also von 
sehr konstanter Größe sein. Eine ebensolche Gleichmäßigkeit der Kurve zeigt sich beim Anstieg der Körpertemperatur infolge der Erhöhung der Außentemperatur.

Nur nach Überschreitung gewisser Extreme der Körpertemperatur sind Anstieg oder Abfall stärker beschleunigt, so daß eine Steigerung, respektive Herabsetzung, auch der Verbrennungen im Organismus zur Erklärung der Kurve herbeigezogen werden muß.

$\mathrm{Da}$ andererseits jede Veränderung der Wärmebildung mit Sicherheit die Körpertemperatur verändert, hatten wir schon bei einer früheren Untersuchung ${ }^{1}$ ) bemerkt. Auf jede Nahrungszufuhr ging die Körpertemperatur unserer Tiere, deren zerebrale Wärmeregulation ansgeschaltet war, sehr erheblich in die Höbe. Das gleiche Verhalten hatten auch Freund und Strasmann ${ }^{2}$ ) bei Tieren mit durchschnittenem Halsmark beschrieben.

Nach Gaben von narkotischen Mitteln, die ja die Gesamtsumme der Verbrennungen im Organismus herabsetzen können ${ }^{3}$, hatten wir die Temperatur prompt und regelmäßig abfallen sehen.

$O b$ und inwiefern die zu prüfenden Gifte bei den Versuchstieren eine Veränderung der Wärmeabgabe hervorrufen können, wird für jede einzelne Substanz zu erörtern sein.

Als allgemeine Bemerkung möchte ich aber folgendes vorausschicken. Die Veränderlichkeit der Gefäßweite, das Spiel der Vasomotoren, welches für die Schwankungen in der Wärmeabgabe unserer Versuehstiere, neben Veränderungen der Körperhaltung, ja im wesentlichen allein in Frage kommt ${ }^{4}$ ), scheint durch unsere operativen Eingriffe aufgehoben oder mindestens in hohem Grade beschränkt zu werden. Schon die große Gleichmäßigkeit des Temperaturverlaufes spricht in diesem Sinne. Auch daß es mir in einer bis jetzt allerdings erst geringen Zahl von Versuchen - ich hoffe bei anderer Gelegenheit darauf zurückkommen zu können - nicht gelungen ist, bei Tieren mit ausgeschaltetem Zwischenhirn durch gefäßverengernde

1) Isenschmid and Krehl, a. a. 0 .

2) a. a. 0 .

3) v. Boeck und Bauer, Über den Einfluß einiger Arzneimittel auf den Gasaustausch bei Tieren. Zeitschr. f. Biol. Bd. 10, 874; E. Müll er, Über den Einfluß von Chloralhydrat und Morphin, Antipyrin, Chinolin und Chinin anf die Kohlensäureausscheidung im tierischen Organismus. Inaug.-Diss. Erlangen 1891; Rumpf, Untersuchungen über die Wärmeregulation in der Narkose und im Schlafe. Pflïgers Arch. Bd. 33, 1884 u. v. a.

4) Walbanm, Arch. f. exper. Path. u. Pharmakol. Bd. 72, 1913, hat kürzlich gezeigt, wie wenig Veränderungen der Atmung die Wärmeabgabe der Kaninchen beeinflussen. 
Mittel Vasokonstriktion hervorzurufen, zeigt mindestens, daß die Beeinflußbarkeit der Gefäßweite reduziert ist. L. R. Müller $\left.{ }^{1}\right)$ nimmt an, daß auch die vasomotorische Innervation, wie die Wärmeregulation, von den an der Basis des Zwischenhirns liegenden, bei unseren Tieren also ausgeschalteten vegetativen Zentren beherrscht wird. Es wäre also nicht verwunderlich, wenn die Gefäßweite unserer Tiere keine Neigung zu Schwankungen zeigte.

Im einzelnen war nnser Verfahren folgendes: Bei Kaninchen wurde der mediane Teil des Hirnstammes hinter dem Zwischenhirn und unmittelbar vor dem vorderen Vierhiugelpaare quer durchtrennt. Die Technik war eine ähnliche, wie wir sie in einer früheren Arbeit ${ }^{2}$ ) beschrieben haben. Geringe, aber nicht unwesentliche Modifikationen der damaligen Technik werden wir später an Hand der genaueren anatomischen Untersuchungen, die ich seit mehreren Monaten gemeinsam mit Herrn Dr. Schnitzler vornehme, darlegen.

In einzelnen Fällen habe ich nach dem Vorgange von Leschke ${ }^{3}$ ) durch einen Stich in jene Gegend die Wärmeregulation auszuschalten versucht. Daß es im Prinzip möglich ist, durch eine solche, wenig ausgedehnte Verletzung die Wärmeregulation vollständig auszuschalten, hoffen wir später an Hand anatomischer Untersuchungen darlegen zu können. Es ist mir auch in einem Falle einwandfrei gelungen.

Die Versuche von Leschke aber, der diese Methode vorgeschlagen und zuerst ausgeführt hat, beweisen vorläufig nur, daß man anf diese Weise die Wärmeregulation mehr oder weniger hochgradig stören kann; ganz aufgehoben war die Wärmeregulation, soweit sich das aus den kurzen Mitteilungen des Autors schließen läßt, bei Leschkes Versuchstieren nicht.

Jene Tiere wurden bei einer Außentemperatur von $16-20^{\circ}$ gehalten und lebten dabei 3-6 Tage, während ihre Körpertemperatur in dieser Zeit ganz langsam und mit Unterbrechungen herunterging, sich im wesentlichen zwischen 33 und $36^{\circ}$ hielt. So verhalten sich meine Tiere nicht, sondern ihre Temperatur fällt bei $18^{\circ}$ innerhalb weniger Stunden auf $30^{\circ}$ und darunter; so daß das Leben in kürzester Zeit erlischt, während sie bei einer Außentemperatur von $26-31^{\circ}$ bis zu 14 Tagen leben können. Meine Tiere lagen fast ausnahmslos anf einer wollenen Decke, so daß ihnen die Unterlage nicht allzuviel Wärme entzogen haben kann.

Die Wärmeregulation könnte ich bei Leschkes Tieren nur dann als vollständig erloschen ansehen, wenn sie in einer Weise gelagert gewesen

1) Kongreß f. innere Medizin 1913, S. 11ó.

2) Isenschmid und Krehl, a. a. 0 .

3) a. a. $O$. und Leschke, Untersuchungen über anaphylaktisches Fieber. Kongreß für innere Medizin 1913; auch Citron und Leschke, Experimentelle Beitrïge zur Frage der Beziehtungen zwischen Nervensystem und Infekt beim Fieber. Ebenda. 
wären, die ihre Wärmeabgabe künstlich beschränkte, wie etwa in einem Nest von Watte, oder wenn sie mehrmals täglich reichlich gefüttert worden wären, so daß die Wärmebildung dadurch so stark gesteigert worden wäre, daß sie bei so niedrigen Außentemperaturen mit dem Leben verträgliche Körpertemperaturen behielten. Daß ein Tier bei einer Temperatur von $20^{\circ}$ seine Körpertemperatur von 31 anf $34^{\circ}$ steigern kann (man vergleiche Kurve Nr. 2 auf Seite 171 der Leschkeschen 1) Arbeit), würde ich wenn nicht etwa Nahrungsanfnahme vorangegangen ist, oder das Tier heftige epileptische Anfälle gehabt hat - als sicheren Beweis für das Bestehen eines guten Restes von Wärmeregulationsvermögen ansehen. Bei dem mächtigen Einfluß, den die Nahrungsaufnahme und die Muskelbewegungen auf die Wärmebildung haben, können wir Beschreibungen von Versuchen, in welchen von beiden nicht die Rede ist, nur schwer deuten. Ich glaube also, daß Leschke durch seinen "Zwischenhirnstich * die Wärmeregulation nur gestört und nicht völlig aufgehoben hat, denn Tiere, die sich verhalten wie die seinigen, habe ich sehr häufig zu beobachten Gelegenheit gehabt, wenn meine Operation wegen Blutungen oder aus einem sonstigen Grunde nicht grïndlich ausgeführt worden war.

Solche Tiere habe ich za den folgenden Versuchen nicht verwandt, sondern nur diejenigen, bei denen das völlige Fehlen der Wärmeregulation durch strenge Prüfung einwandfrei festgestellt worden war. Die Prüfung bestand darin, daß die Tiere im Hunger in eine Temperatur von $18-20^{\circ}$ gebracht wurden. Wenn bei stündlicher Messung die Temperatur gleichmäßig abfiel, so daß das Tier innerhalb drei bis funf Standen eine Temperatur von unter $35^{\circ}$ zeigte, wurde es zunächst für wahrscheinlich gehalten, daß die Regulation erloschen war. War dagegen der Abfall kein ganz gleichmäßiger, so z. B., daß das Tier in einer Stunde um $1,2^{\circ}$ an Temperatur verlor, während es in der nächsten Stunde nur um 0,5 oder $0,6^{\circ}$ abfiel, wurde es für wahrscheinlicher gehalten, daß ein kleiner Rest von Wärmeregulationsvermögen doch noch erhalten war. Das Tier wurde deshalb länger bei Zimmertemperatur gehalten. Kam der Abfall nach einigen Stunden zum Stillstand, oder hob sich dabei gar die Temperatur um einige Zehntel, wurde angenommen, daß das Tier noch einen Rest von Wärmeregulation besaß.

Hatte sich ein Tier einwandfrei abgekühlt, wurde es in einen Thermostaten von etwa $30^{\circ}$ gebracht, gewöhnlich außerdem auf einige Stunden in Watte eingepackt, bis es eine Temperatur zwischen 38 und $40^{\circ}$ aufwies und dann mehrere Stunden hindurch festgestellt, bei welcher Temperatur des Wärmeschrankes die Körpertemperatur konstant bleibt. Dann wurde der Wärmeschrank um $2-3^{\circ}$ niedriger eingestellt und verlangt, daß das Tier prompt mit seiner Körper-

1) Zeitschr. f. exper. Path. u. Therap. Bd. 14, S. 167. 
temperatur herabging. Diese zwei Versuche müssen unbedingt gemacht werden, ehe eine vollständige Aufhebung der Wärmeregulation angenommen wird. Auf Versuche, die Tiere durch Verbringen in wärmere Umgebung zu überhitzen, kann dagegen verzichtet werden. Einmal sind solche Versuche für die Tierc gefährlich, besonders deshalb, weil am Gehirn operierte Kaninchen bei Körpertemperaturen von über $40^{\circ}$ oft zu epileptischen Anfällen neigen ${ }^{1)}$. Ferner überbitzen sich einzelne normale Tiere bei längerer Einwirkung bei den gleichen Außentemperaturen, die wir anwenden müßten, um unsere operierten Tiere auf über $40^{\circ} \mathrm{zu}$ bringen. Die Probe wïrde also oft nicht viel beweisen. Dagegen ist die Temperatursteigerung nach der Nahrungsanfnahme, ferner diejenige, welche, wie ich noch zeigen werde, auf kleine Salizyldosen eintritt, für die Tiere obne Wärmeregulation charakteristisch ${ }^{2}$ ).

Alle Versuche, die ich an Tieren mit durchtrenntem Hirnstamm machte, wiederbolte ich mehrmals an Tieren, deren Wärmeregulation nach Freund und Strasmann ${ }^{3}$ ) durch Quertreunung des Ruickenmarkes (gewöhnlich am sechsten Halswirbel) ausgeschaltet war. Ist die Trennung technisch einwandsfrei gelungen, und darüber ist man nach der Operation selten im Zweifel, ist eine genanere Prïfung des Wärmeregulationsvermögens hier nicht notwendig, denn es ist regelmäßig völlig erloschen.

Die Versuche mit den Giften wurden folgendermaßen ausgeführt: Die Kaninchen, deren Wärmeregulation völlig aufgehoben war, wurden in einen Brutschrank gebracht, dessen Temperatur so eingestellt war, daß die Körpertemperatur des Tieres dabei von normaler Höhe (gewöhnlich zwischen 38 und $39,5^{\circ}$ ) war und absolut konstant blieb. Wenn mehrere Stunden hindurch die Körpertemperatar keine Schwan-

1) Die Neigung zu Krampfanfüllen bei hoher, und das Aufhören der Anfülle bei niedrigerer Temperatur ist also keine Besonderheit der Tiere ohne Schilddrüsenapparat, wie Boldyreff, Pfïgers Archiv Bd. 154, 1913 glaubt, sondern wohl eine Tatsache von allgemeinerer Geltung. Den Kinderïrzten ist bekannt, daß Frühgeburten, deren Wärmeregulation schlecht entwickelt ist, bei künstlicher Überhitzung leicht Krämpfe bekommen.

2) Ich lege auf eine eingehende Prïfung des Wärmeregulationsvermögens besonders auch deshalb Gewicht, weil in allerletzter Zeit auch von einer weiteren Seite Tiere mit mehr oder weniger gestörter Wärmeregulation den Tieren mit fehlender Wärmeregulation gleichgestellt worden sind (Boldyreff in der oben angefïhrten Arbeit über Tiere mit exstirpiertem Schilddriisenapparat). Wenn Störung and $\Delta$ ufhebung der Wärmeregulation nicht sorgfältig anseinandergehalten werden, besteht für die Znkunft die Gefahr einer großen Verwirung, denn es ist wahrscheinlich, daß noch durch manche verschiedene Eingriffe am tierischen Organismus die Wärmeregulation mehr oder weniger gestört werden kann. 3) a. a. 0. 
kungen von melr als zwei Zehnteln gemacht hatte, wurde das Gift, gewöhnlich subkutan, seltener per os oder intravenös verabreicht und die Temperatur des Tieres auch weiter, gewöhnlich stündlich, gemessen. Die Temperatur des Ofens wurde wäbrend des ganzen, mindestens einen halben Tag dauernden Versuches jede halbe Stunde kontrolliert, denn es muß verlangt werden, daß der Ofen keine Schwankungen von mehr als ein Drittel Grad macht. Bei sorgfältig geleiteten Versuchen and einwandfreien, $\mathbf{d}$. h. besonders keine spontanen Muskelbewegungen ausführenden Tieren ${ }^{1)}$ sind Schwankungen von drei Zehntel Graden und mehr mit Sicherheit zu verwerten, d. b. auf die Wirknng des geprüften Giftes zu beziehen. Schwankungen bis zu drei Zehnteln liegen auch bei guten Versuchen innerhalb der Fehlergrenze.

Die Tiere waren während des Versuches im Hnngerzustand. Da in der Regel täglich ein Versuch gemacht wurde, und vom dritten Tage nach der Operation an ebenfalls alle 24 Stunden gefüttert wurde, mußte die Fütterung knapp sein und aus leicht resorbierbaren Stoffen bestehen. Das gewöhnliche Pflanzenfutter kam dafür nicht in Betracht, da dessen Wirkung auf den Stoffwechsel viel zII Iange anhält. Findet man doch noch zwei bis drei Tage nach der Aufnahme von Grünfutter bei Sektionen reichliche Reste im Magen und Darm. Rubner ${ }^{2}$ hat für das Meersehweinchen nachgewiesen, daß diese Speisereste kalorisch gar nicht geringwertig sind. Wir wäblten als leicht resorbierbare Nabrung Haferschleim mit einem geringen Zusatz vou Eidotter, der meistens in einer Portion $(80-150 \mathrm{ccm})$ per Schlundsonde verabreicht wurde. Am Halsmark durchschnittene K.aninchen nehmen dieses Futter auch spontan zu sich, wenn man es diesen gelähmten Tieren bequem hinhält. Tiere mit ausgeschaltetem Zwisehenhirn, die spontan nicht fressen, kann man mindestens durch Einspritzen des Futters in den hinteren Teil der Mundhöhle sehr leicht reflektorisch zum Schlucken bringen und ihnen so bis über $200 \mathrm{ccm}$ in kurzer Zeit beibringen. Die Fütterung mit der Schlnndsonde wurde aber als weniger zeitraubend meistens vorgezogen. Schon 12-15 Stunden aach einer solchen Fätterung war ihr den Stoffwechsel steigernder Einfluß bei unserer Vexsuchsanordnung in der Regel nicht mehr nachweisbar, so daß wir also die Tiere als hungernd betrachten durften, und dies sogar mit besserem Recht, als man es etwa bei Tieren kann, welche nach reichlicher Aufuahme von Hafer und Rüben 36-40 Stunden gehungert haben, denn bei solchen

1) Die meisten auch der am Gehirn operierten Tiere machten sehr wenige, oft halbe Tage lang gar keine Bewegangen. Dadurch waren sie zu Untexsuchungen der Würmebildung a. dgl. viel brauchbarer als normale Tiere. Einzelne Kaninchen zeigten infolge von Reizerscheinungen im Gehirn allerlei Bewegungen, z. B. Reitbahnbewegungen, rhythmische Bewegungen der Extremitäten u. dgl. Selbstverständlich blieben solche Tiere, wie auch solche, welche schon bei norwaler Körpertemperatur epileptische Anfälle zeigten - bei hohen Temperaturen von $40-41^{\circ}$ kamen solche fast regelmäßig vor - von unseren Vergiftungsversuchen abgesehen.

2) a. a. 0 . 
Tieren haben wir noch öfter Unregelmäßigkeiten der Temperatur beobachtet, besonders eine Neigung za Steigerungen, die wir uns nur dadurch erklären konnten, daß doch immer noch ein Einfuß der Nahrung bestand.

Die Nahrung, die wir zuführten, reichte bei weitem nicht aus, um den 24 stiundigen Kalorienbedarf der Tiere zu decken. Sie waren also, auch abgeseben vom Hunger während der Versuche, stark unterernährt.

\section{Natrium salicylicum.}

Über die Wirkung der Salizylsäure auf die Wärmeproduktion und den Gesamtstoffwechsel sind wir auffallenderweise sehr wenig gut unterrichtet.

Daß die Stickstoffausscheidung nach der Aufnahme von Salizylsäure fast ausnahmslos stark gesteigert ist, dafur finden sich in der Literatur zahlreiche, übereinstimmende Belege ${ }^{1}$ ). Die Wirkung auf den Gasaustansch und auf die direkt gemessene Wärmeproduktion ist aber immer nur in wenigen Versuchen, oft nur nebenbei, untersucht worden. In den besten neueren Lehrbüchern der Arzneimittellehre wird sie, soweit davon überhaupt die Rede ist, gewöhnlich gemeinsam mit der Wirkung der Substanzen der Antipyringruppe abgehandelt und in dieser Hinsicht als damit wesensgleich hingestellt ${ }^{2}$ ). Den letzteren mißt man aber im allgemeinen einen, wenn auch geringen, steigernden Einfluß auf den Gesamtstoffwechsel bei. Gottli $\mathrm{b}^{3}$ ) bezeichnet die Wirkung der Salizylsäure auf den Wärmehaushalt als ungenügend studiert. Anch Loewi und Weber drücken sich in ihren zusammenfassenden Darstellungen ${ }^{4}$ ) darüber recht zurückhaltend ans; Weber gibt an, daß bald Herabsetzung, bald Steigerung des Gasumsatzes je nach der Dosis gefunden wurde. Loewi führt vor allem die Singersehen Versnche an, in denen auf kleine Dosen Aspirin eine Herabsetzung, anf große eine Steigerung der 0-Ausscheidung festgestellt warde. Außer diesen zwei Versuchen von Singer ${ }^{5}$ ) habe ich nur noch in einem Ver-

1) Kumagawa, Virch. Areh. Bd. 113, 1888: C. Virchow, Über die Einwirkung des benzoesauren und des salizylsanren Natrons auf den Eiweißumsatz in Körper. Hoppe-Seylers Zeitsehr. f. physiol. Chemie Bd. 6, 1882; Le e or ché, Revue mensuelle de Med. 1880 zit. nach Goodbody; Goodbody, The influence of Sodinm salicylate on general metabolism. Journ. of Physiol. Bd. 25, 1900, S. 399 u. v. a.

2) So in Schmiedebergs Grundriß der Pharmakologie, 7. Auflage, Leipzig 1913.

3) In H. Meyer und Gottlieb, Die experimentelle Pharmakologie usw. Berlin und Wien 1910.

4) Loewi in v. Noordens Handbuch der Pathologie des Stoffwechsels. II. Bd., II. Auflage, Berlin 1907; Weber, S., Über die Beeinflussung des Stoffwechsels durch einige pharmakologisch wichtige Stoffe. Ergebnisse der Physiologie III, I.

5) Über Aspirin. Beitrag zur Kenntnis der Salizylwirkung. Pfügers Arch. Bd. 84, 1901, S. 527. 
suche von Livon') die Angabe finden können, daß der Gesamtstoffwechsel (hier die $\mathrm{CO}_{2}$-Ausscheidung) auf kleine Dosen Salizyl, $, 0,02-0,03$ bei inem Meersehweinchen) herabgesetzt war. Auf größere Dosen, und hierin besteht recht gute Übereinstimmung zwischen den verschiedenen Untersuchern, hat anch dieser Beobachter Steigerung der Gasausscheidung gefunden. Stühlinger ${ }^{2}$ ) hat in sechs Versuchen mit mittleren und größeren Dosen $(0,1-0,225)$ bei Meerschweinchen zweimal ein Gleichbleiben, viermal eine dentliche Steigerung der Wärmeproduktion beobachtet. Besonders interessant schien mir der Versuch mit der höchsten Dosis zu sein. Hier stieg die Wärmebildung im Verbältnis von $100: 182$, die Temperatur des Tieres auf $41,4^{\circ}$. Hier ist die Wärmeregulation durch das Gift und die dadurch bedingte Steigertung der Verbrennungen sozusagen über den Haufen gerannt worden, so daß das Tier sich ähnlich verhielt wie meine Kaninchen ohne Wärmeregulationsvermögen.

Mit Natrium salicylicum labe ich zehn verschiedene Versuche gemacht. In allen ohne Ausnahme trat eine sehr erhebliche Temperatursteigerung ein.

Die ersten Versuche wurden an Tieren mit Gehirnoperation und mit hohen Dosen $(0,5$ pro Kilo Tier $)$ vorgenommen. In zwei Versuchen spritzte ich eine lösung von holer Konzentration (33\%) subkutan ein. Die Einspritzung verursachte anscheinend Schmerzen und die Tiere waren deshalb während einiger Minuten so unruhig, daf ihre Wärmeproduktion dadurch gesteigert gewesen sein mag. Ich wählte deshalb zu allen späteren Versuchen eine diunnere, 2,5\% ige, ungefähr isotonische, oder eine 5\% ige Lösung, auf die keine oder nur ausnabmsweise sebr geringe Schmerzensänßerungen in Erscheinung traten. Die Temperatursteigerung fiel nicht geringer aus.

In den vier Versuchen mit der Dosis von $0,5^{3}$ ) bekam ich Temperatursteigerungen von $1,05^{\circ}$ beim ersten Tier, das zweite starb eine Stunde nach der Einspritzung, nachdem sich seine Temperatur um 1,10 erböht hatte. Ein anderes Tier bekam, nachdem seine Temperatur in $21 / 2$ Stunden um 2,40 auf 41,4 gestiegen war, einen schweren Krampfanfall, an dem es einging; ein viertes Kaninchen zeigte eine Steigesung um $3,25^{\circ}$ auf 41,75 . Um das Tier nicht zu verlieren, brach ich den Versuch $a b$, indem ich es in eine niedrige Anßentemperatur brachte. Auf solehe hohe Dosen waren alle Tiere sehr erregt, die Frequenz der Atmung oft anf das dreifache gesteigert, der Muskel-

1) Comptes rendus de l'Académie des seiences Paris Bd. 90, 1880.

2) Über die Einwirkung einiger antipyretischer Mittel auf den Wärmehaushalt gesander und kranker Tiere. Arch. f. exper. Path. u. Pharmakol. Bd. 43, 1900.

3) Meine Angaben iiber die Dosen beziehen sich, wenn nichts anderes bemerkt ist, immer auf das Kilo Körpergewicht. 
tonus erhöht, manche Tiere machten vielerlei Bewegungen, so daß ich geneigt war, anzunehmen, daß die beobachtete Steigerung der Wärmeproduktion nur eine Folge dieser verstärkten Muskelaktion war. Ich wiederholte deshalb den Versuch an Tieren mit durchschnittenem Halsmark, die ja, abgesehen vom Zwerchfell und der Muskulatur des Halses und des Kopfes, gelähmt sind, bei welchen also Muskelbewegungen nur wenig Einflaß auf den Gesamtstoffwechsel haben konnten und war überrascht zu sehen, daB die Temperatursteigerung auf Salizyl keineswegs geringer ausfiel. Ein solches Tier bekam z. B. auf 0,4 Natrium salicylicum eine Steigerung ron $2,2^{\circ}$, während ein weiteres, am Gehirn operiertes Tier auf eine nur wenig geringere Dosis $(0,35)$ nur eine Steigerung von 1,35 bekam. (Die Kurven der beiden letztgenannten Tiere sind in Fig. 1 und 2 wiedergegeben.)

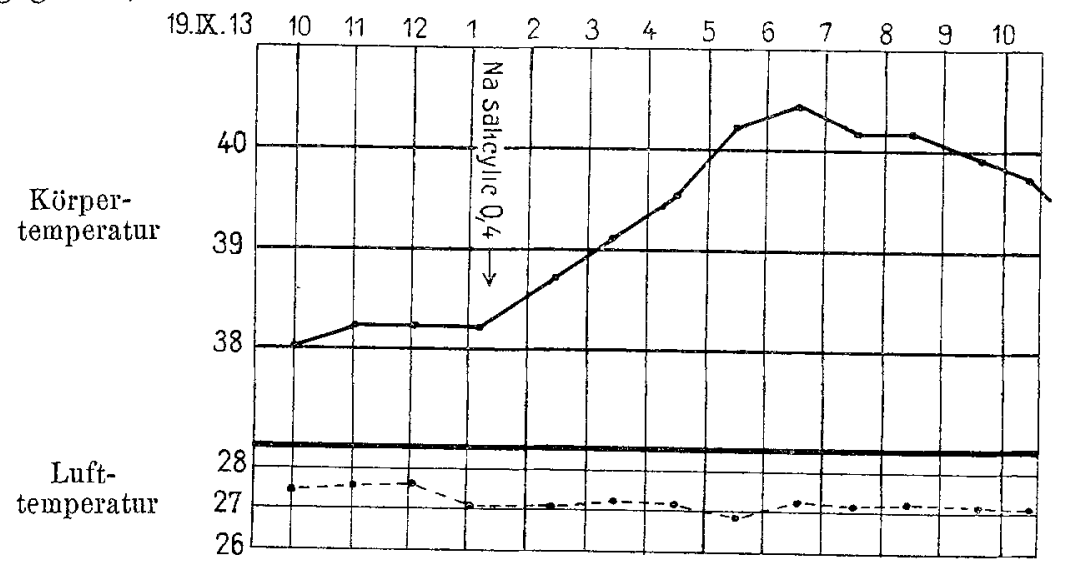

Fig, 1. Wirkung von $0,4 \mathrm{~g}$ Nati, salicylic. auf ein Tier mit durchschnittenem Halsmark.

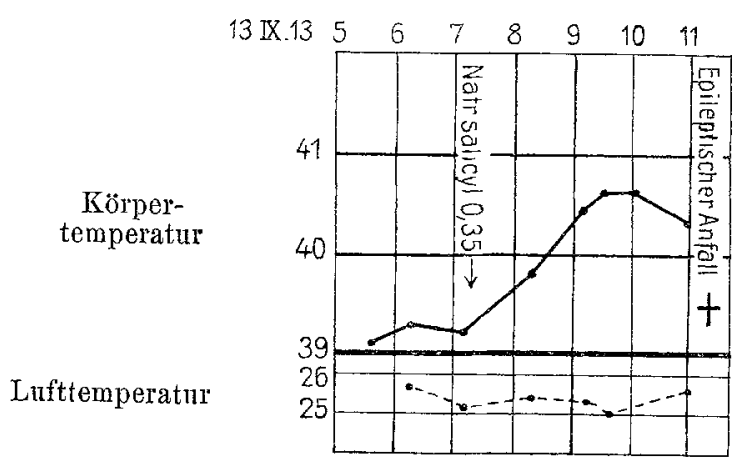

Fig. 2. Wirkung von $0,35 \mathrm{~g}$ Natr. salicylic. auf ein Kaninchen mit ausgeschaltetem $Z$ wischenhirn. 
Im Hinblick auf die angeführten Versuche von Singer 1 ), welche ja bei niedrigen Dosen von Aspirin eine Redultion des Sauerstoffkonsums um 17 und 14\% ergeben hatten, gab ich in Form ron Natrinm salicylicum bei drei Tieren ebenso kleine Dosen (auf das Kilo Tier berechnet), wie sie Singer gegeben batte, nämlich 0,11 und 0,13 per os bei zwei Tieren mit durchschnittenem Rüekenmark und 0,12 subkutan bei einem im Gehirn operierten Tiere. Alle zeigten sie einen deutlichen Ausschlag von $+0,45 ;+1,65$ und $+1,1^{\circ}$. Bei der Darreichung per os, die ich wählte, um meine Versuche mit denjenigen von Singer, der das Medikament auch auf diese Weise gab, leichter vergleichen zu können, trat die Steigerung entsprechend der langsameren Resorption etwas langsamer ein, so daß der Gipfel der Kurve das eine Mal nach drei, das andere Mal erst nach 9-10 Stunden erreicht wurde, während bei subkutaner Darreichung der Anstieg so raseh erfolgte, daß der Gipfel der Kurve gewöhnlich in die 2. oder 3. Stunde nach der Einspritzung fiel. Als zehnten Versuch habe ich einen mit der Dosis von 0,17 pro Kilo bei einem Tier mit durchschnittenem Halsmark anzuführen. Die Steigerung betrug 1,0 ${ }^{\circ}$.

Bei den kleineren Dosen fand sich bei den nicht gelähmten Tieren und in den nicht gelähmten Muskelpartien der Tiere unit durehnittenem Riickenmark in einzelnen Fällen keinerlei Steigerung des Muskeltonus, spontane Bewegungen fehlten völlig, in zwei Fällen fehlte auch die Steigerung der Atemfrequenz und trotzdem trat eine ebenso starke Steigerung der Temperatur ein wie bei den Tieren, welche Zeichen von Erregung darboten.

Daß die Steigerung der Temperatur durch eine Beschränkung: der Wärmeabgabe zu erklären wäre, dafür haben wir keinerlei Anhaltspunkte. Es ist nicht bekannt, daB Salizylsäure eine Verengerung der Hautgefäße erzeugen könnte, sondern eher das Gegenteil zu erwarten. Aus den in der Einleitung erwähnten allgemeinen Grïnden ist es mir wahrscheinlicher, $d a ß$ die Hautgefäße durch das Gift in ihrer Weite überhaupt nicht wesentlich beeinflußt waren.

Ich $m u B$ aus diesen Versuchen also schließen, daß die Salizylsäure bei Kaninchen ohne Wärmeregulation die Gesamtsummeder Verbrennungen immer, anch in kleineren Dosen, erbeblicb steigertund zwar unabhängig von einer etwa auftretenden Steigerung der Motilität.

Ob das entgegengesetzte Ergebnis der Singersehen Versnche sich dadurch erklärt, daß bei kleinen Dosen die priminre steigernde Wirkung 
der Salizylsäure etwa durch die chemische Wärmeregulation überkompensiert wurde, muß vorläufig dahingestellt bleiben. Es besteht anch die Möglichkeit, daß während der Untersuchung des Gaswechsels (50-90 Minuten und 60-150 Minuten nach der Einführung per Schlundsonde) die Tiere noch gar nicht unter Salizylwirkung standen. Aspirin zerfallt ja im Magen kaum ${ }^{1}$, und da der Kaninchenmagen bekanntlich sehr zur Speicherung seines Inhaltes neigt, waren zur Zeit der Singerschen Beobachtungen vielleicht noch keine nennenswerten Mengen resorbiert.

\section{Antipyrin.}

Über den Einfluß des Antipyrins auf den Energieumsatz besitzen wir sehr gute Untersuchungen. Gottlieb ${ }^{2}$ ) fand auf Dosen von 0,5 bei normalen Kaninchen ron durchschnittlich $2 \mathrm{~kg}$ Gewicht eine Steigerung der Warmeproduktion $u$ m $5-8 \%$, bei größeren, stark toxisch wirkenden Gaben beobachtete er Herabsetzung der Wärmebildung. Eine ähnliche steigernde Wirkung auf die Wärmeproduktion fand auch bei Tieren nach dem Wärmestich statt. Der Autor vermutet, daß die Steigerung der Wärmebildung anf regulatorischem Wege als Reaktion auf die durch das Arzneimittel gesteigerte Wärmeabgabe zustande kommt. In gleicher Weise faßt der Autor die Erscheinungen auch in einer neueren Darstellung ${ }^{3}$ ) auf. Auch Stühlinger ${ }^{4}$ fand bei gesunden Kaninchen in der Regel eine Steigerung der Wärmebildung auf Gaben von $0,5 \mathrm{~g}$ Antipyrin. In einzelnen Fällen blieb die Wärmebildung unbeeinflußt. Bei Meerschweinchen sank die Wärmeproduktion anf Antipyrin gewöhnlich. Bei fiebernden Kaninchen sank sie in der Regel. Auch Stühlinger bezieht diese Wirkung nicht auf einen direkten Einfu $\beta$ des Giftes auf die Stätten des Stoffwechsels, sondern er nimmt an, daf die wärmeregulierenden Zentralapparate sie vermitteln. Riethus ${ }^{5}$ fand in einem Falle bei einem nicht fiebernden Erysipelkranken eine geriuge Herabsetzung des Gaswechsels auf Antipyrindarreichung. Ebenso sah Liepelt ${ }^{6}$ ) an gesnnden Menschen auf Autipyrin bald eine geringe Herabsetzung, bald ein Gleichbleiben des Gasumsatzes. Auf ältere, methodisch weniger gute Untersuchungen des Energieumsatzes nach Antipyrin soll hier nicht eingegangen werden, auch lasse ich weitere Versuche an fiebernden Individuen bei Seite, dagegen haben wir die Versuche von Krehl und Matthes ${ }^{7}$ ) anzufuhren, die bei Kaninchen mit durch-

1) Dreser, Pharmakologisches iuber Aspirin. Pfïgers Archiv Bd. 76, 1899.

2) Kalorimetrische Untersuchungen über die Wirknngsweise des Chinin und Antipyrin. Arch. f. exper. Path. u. Pharmakol. Bd. 28, 1891.

3) H. Meyer und Gottlieb, Die experimentelle Pharmakologie usw. Berlin-Wien 1910, S. 393.

4) a. a. 0 .

5) Beobachtungen über den Gaswechsel kianker Menschen und den Einfluß antipyretischer Medikamente anf denselben. Arch. f. experiment. Pathol. und Pharmakol. Bd. 44, 1900.

6) Der EinfluB von Antipyrin und Chinin auf den Gaswechsel des gesunden Menschen. Arch. f. experiment. Pathol. und Pharmakol. Bd. 43, 1900.

7) zit. bei Stühlinger a. a. 0. S. 187. 
schnittenem Halsmark, welche bei $27^{\circ}$ anf ihren Wärmehaushalt nntersucht wurden, anf Antipyrin keine Veränderung der Wärmebildung und Wärmeabgabe beobachteten.

Ich verfüge über 13 Versuche mit Antipyrin. Davon sind vier nicht zu verwerten, weil entweder der Brutschrank oder das Tier sich unregelmäßig verhielten. Von den neun vollwertigen Versuchen betreffen vier Tiere mit durehsehnittenem Halsmark. Die Dosen waren $0,23,0,26,0,4$, und 0,4 in subkutaner Darreichung. Keines von den Tieren zeigte - in striktem Gegensatze zu den Versuchen mit Salizyl - eine Veränderung seiner Temperatur. Von den fünf Versuchen an Tieren mit durchtrenntem Hirnstamm fand sich in einem ebenfalls kein Ausschlag, während in den vier anderen in den ersten Stunden nach der Einspritzung die Temperatur deutlich in die Höhe ging. Die Steigerung war bei den angewandten Dosen viel kleiner als die auf Salizyl stattfindende. Sie betrug $\left.0,6(0,34)^{1}\right), 0,7(0,25)$, $0,7(0,5)$ und im vierten Falle, in dem der Wärmeschrank vielleicht um einige Zehntel zu hoch gestanden hat, $1,95^{\circ}(0,5)$. In allen Versuchen fiel eine Veränderung der Motilität der Tiere auf. Besonders war der Muskeltonus bei den höheren Dosen deutlich gesteigert, die meisten Tiere bewegten sich auch spontan, gewöhnlich war auch die Frequenz der Atmung gesteigert, so daß angesichts des Fehlens der Steigerung bei den gelähmten Tieren die Vermutung nahe liegt, daß, im Gegensatz z nalizyl, die auf Antipyrin nicht regelmäßig und nur bei nicht gelähmten Tieren auftretende Steigerung der Wärmebildung eine Folge der veränderten Muskelinnervation ist.

Daß eine Verminderung der Wärmeabgabe die Steigerung der Temperatur bedingt haben sollte, ist sehr unwahrscheinlich angesichts der vielen Erfahrungen, die eine entgegengesetzte Wirkung des Antipyrins dartun. DaB das Antipyrin auch bei unseren Tieren die Wärmeabgabe gesteigert haben könnte und dadurch die Ausschläge beeinflußt hätte, ist nicht sicher auszuschließen, aber aus früher dargelegten Gründen nicht eben wahrseheinlich.

$\mathrm{Ob}$ bei nicht operierten Tieren die Steigerung der Wärmebildung wirklich, wie Gottlieb und Stühlinger annahmen, durch Vermittlung der Wärmeregulationszentren zu. Stande kommt, ist auf Grund unserer Versuche zwar nicht auszuschlieBen, aber es könnte doch auch bei normalen Tieren eine Steigerung der motorischen Innervation, welche $z u$ ihrem Zustandekommen keinerlei Wärmeregulationszentren bedarf, zur Erklärung herbeigezogen werden.

1) Die Zahl in der Klammer gibt die Dosis an. 


\section{Chinin.}

Die sehr zahlreichen Untersuchungen, die wir über die Wirkung des Chinins auf den Stoffumsatz nicht fiebernder Warmblüter besitzen, haben zu wiedersprechenden Resultaten gefuhrt. In der Regel, wenn auch nicht ausnahmslos ${ }^{1}$, wurde allerdings eine Herabsetzung ${ }^{2}, 3$ ) der Stickstoffausscheidnng konstatiert, manchmal nach einer anfänglichen Steigerung ${ }^{4}$ ).

In der Größe der Wärmebildung und des Gasumsatzes wurde dagegen nach mäßigen Chinindosen mindestens ebensooft wie eine Herabsetznng $\left.{ }^{5},{ }^{6}, 7\right)$ ein Gleichbleiben ${ }^{8}, 9,10$ ) oder gar eine Steigerung ${ }^{11},{ }^{12}, 13$ ) gefunden. Die Steigerung kam bei hohen Dosen öfter vor als bei niederigen.

Angesichts der lähmenden Einwirkungen des Giftes auf manche einzellige Organismen und auf die Leukocyten hat die Annahme einer primären, in den Geweben selbst angreifenden, den Stoffwechsel herabsetzenden Wirkung des Chinins seit langem ein starkes Argument für sich gehabt und ältere Versuche an Tieren mit Läsionen des Zentralnervensystems unterstützten diese Annahme. Die Vermutung von Loewi ${ }^{14}$ ), nach der die primäre Herabsetzung des Stofiwechsels durch die chemische Wärmeregulation kompensiert würde; also die geringere Verbrennung von Eiweiß durch eine vermehrte Verbrennung von stickstofffreiem Material oft wettgemacht würde, könnte die beobachteten Tatsachen in befriedigender Weise erklären. Es ist also von Interesse, festzustellen, wie sich der Umsatz nach Ausschaltung des zentralen Regulationsmechanismus gestaltet. Wir besitzen schon mehrere darauf gerichtete Untersuchungen. Lewitzky ${ }^{15}$ ) sah, daß Kaninchen mit durchschnittenem Rückenmark auf - außerordentlich hohe

1) H. Oppenheim, Beiträge zur Phys. und Pathol. der Harnstoffausscheidung, Pflügers Arch. Bd. 23, 1880.

2) Kumagawa, Virchows Arch. Bd. 113, 1888.

3) Prior, Über den Einfluß des Chinins auf den Stoffwechsel des gesunden Organismus, Pflỉgers Arch. Bd. 34, 1884.

4) v. Noorden und Zuntz, Über die Einwirkung des Chinins auf den Stoffwechsel des Menschen. Areh. f. Anat. und Physiol. 1894.

5) v. Boeck und Baner a. a. O.

6) Stühlinger a. a. 0 .

7) Gottlieb a. a. 0., Arch. f. experiment. Pathol. nnd Pharmak. Bd. 28. Man vergleiche damit anch die Deutnng, die Loewi in $\checkmark$. Noordens Handbuch der Pathol. des Stoffwechsels Bd. 2 den Gottliebschen Versuchen gibt.

8) Arntz, Über den Einfluß des Chinins auf Wärneabgabe und Wärmeproduktion. Pflügers Arch. Bd. 31, 1883.

9) Riethus a. a. 0 .

10) E. Mü ülex a. a. 0 .

11) v. Bock und Baner a.a. O.

12) Stiihlinger a. a. 0.

13) Liepelt a. a. 0 .

14) Loewi, Ergebnisse der Physiologie III. 1. 1904, und in v. Noordens Handbuch a. a. 0.

15) Über den Einfluß des sehwefelsatren Chinins auf die Temperatur und Blutzirkulation. Virchows Arch. Bd. 47, 1869. 
- Chinindosen sich rascher abkühlten als im unvergifteten Zustande. Naunyn und Quincke ${ }^{1}$ fanden, daß Honde mit durchschnittenem Halsmark sich in einem sehr warmen Wärmekasten nach Einverleibung von Chinin etwas weniger rasch überhitaten als ohne dasselbe, und $\mathrm{Binz}^{2}$ ) kam mit einer ähnlichen Versuchsanordnung zu einem ähnlichen Ergebnis. Diese dem damaligen Stande der Technik entsprechenden älteren Versuche machen eine weitere Prüfung der Frage mit neneren Hilfsmittelı nicht überllüssig. Wir haben allerdings ans neuerer Zeit eine knrze Angabe über Versuche von Krehl und Matthes ${ }^{3}$ ) an Kaninchen mit durchschnittenem Halsmark. Im Brutkasten wurde ihre Wärmeabgabe und Warmebildung bestimmt und nach Applikation von Chinin beide nicht unbeträchtlich herabgesetzt gefunden, Die näheren Einzelheiten dieser Versuche sind anscheinend nie mitgeteilt worden.

In 19 Versuchen mit subkutanen Injektionen von salzsaurem Chinin bekam ich dreimal keinen Ausschlag, zwölfmal eine Herabsetzung, viermal eine Steigerung der Temperatur. In einem dieser letzteren Fälle war die Steigerung offensichtlich die Folge eines epileptischen Anfalles, in einem zweiten war das Tier motorisch sehr erregt, es setzte seine Wärmeabgabe außerdem während des Versuches noch herab dadurch, daB es von der bisher eingenommenen liegenden Stellung in die normale hockende Haltung iiberging. In den zwei übrigen Versuchen, die za meinen ersten Beobachtungen gehörig in eine Zeit fallen, in der ich auf das motorische Verhalten der Tiere nicht planmäßig achtete, fịde ich darüber überhaupt keine Notizen. Das eine von diesen Tieren stand aber unter der Wirkung der hohen Dosis von 0,2 pro Kilo. Auf solche Dosen habe ich später eine Steigerung des Muskeltonus und eine gewisse motorische Unruhe sowohl bei nicht gelähmten operierten Tieren wie auch bei normalen Kaninchen nie vermißt.

In der großen Mehrzahl der Versuche, bei denen eine Herabsetzung der Temperatur auftrat, waren Dosen von 0,12 bis 0,166 einverleibt worden, ja, unter den acht Tieren, die mit einer Dosis von dieser Höhe behandelt worden waren, findet sich kein einziges, welches keine Herabsetzung der Temperatur aufzuweisen hätte. Unter den drei Tieren, die keinen Ausschlag zeigen, finden sich zwei mit kleineren Dosen behandelte Tiere mit durchschnittenem Halsmark $(0,1$ und 0,081 ) und als drittes ein Tier mit der Dosis von 0,17 , welches starke motorische Erregung zeigte und unmittelbar nach AbschluB des

1) Über den Einfluß des Zentralnervensystems auf die Wärmebildung im Organismus. Areh. f. Anat und Physiol. 1869, S. 175 und 527.

2) Über die antipyretische Wirlsung von Chinin und Alkohol. Virchows Areh. Bd. 51, 1870.

3) a a. 0 . 
Versuches an einem epileptischen Anfall einging. Der Abfall der Temperatur bei den am meisten angewandten mittleren Dosen betrug $0,3-2,05^{\circ}$; durchschnittlich und am häufigsten betrug die Senkang 0,55 bis $0,8^{\circ}$. Der Tiefpunkt der Kurve lag jedesmal in der 3. oder 4. Stunde nach der Einspritzung (vergleiche dazu Fig. 3). Höhere Dosen sowohl als niedrigere zeitigten, wie schon gesagt, hänfig entweder keinen Ausschlag oder einen Ausschlag in der entgegengesetaten Richtung oder aber jedenfalls eine geringere Herabsetzung der Temperatur als die mittleren Dosen.

Daß die hohen Dosen deshalb nicht herabsetzend wirkten, weil sie die motorische Innervation der Tiere zu stark steigerte, ist für

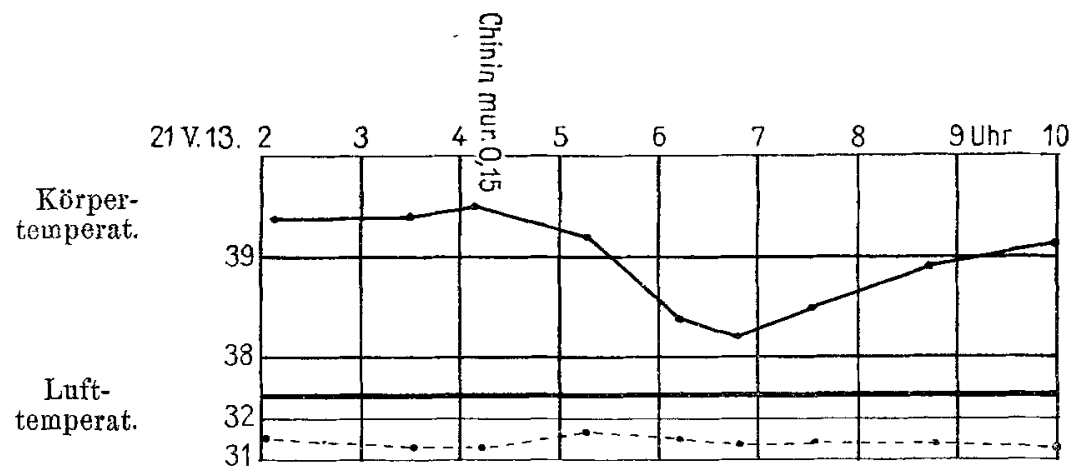

Fig. 3. Wirkung von 0,15 Chinin. muriat. auf ein Tier mit durchschnittenem Hirnstamm.

mich kaum zweifelhaft. Auch würde wahrscheinlich manches der mit mittleren Dosen behandelten Tiere eine erheblichere Temperatursenkung erfahren haben, wenn seine Bewegungen und sein Muskeltonus nicht durch das Gift gesteigert worden wären. Leider verfugge ich zum Vergleich nur über ein Tier mit durchschnittenem Rückenmark. Sein Ausschlag von $0,55^{\circ}$ auf eine Dosis ron 0,16 war allerdings nicht größer als derjenige der nicht gelähmten Tiere. Drei andere Tiere mit durchschnittenem Halsmark bekamen sebr kleine Dosen und dementsprechend kleine oder gar keine Ausschläge.

Ob das Chinin bei meinen Tieren nicht vielleicht auch die Wärmeabgabe vermindert hat, so daß die Temperatursenkung dadurch verkleinert worden ist, ist nicht auszuschließen. Daß aber eine Abnahme der Wärmeproduktion, wenn auch vielleicht nicht quantitativ, in den angeführten Versuchen zum Ausdruck kommt, dürfte nicht zweifelhaft sein. 
Ich schließe also: Die Ansicht, daß Chinin eine vom zentralen Wärmeregulationsmechanismus unabhängige Herabsetzung der Verbrennungen hervorruft, ist richtig. Diese Herabsetzung wird, besonders bei höheren Dosen, durch die durch das Chinin hervorgerufene Steigerung der Motilität mehr oder weniger vollständig kompensiert, gelegentlich auch in eine Steigerung umgewandelt.

\section{Morphin.}

Die Einwirkung des Morphins auf die Wärmebildung ist in den in der Literatur niedergelegten Versuchen ein änßerst verschiedener, je nach der Dosis und dem Versuchstier.

Auf Katzen, die auf das Gift sehr leicht mit Krämpfen reagieren, fanden von Boeck und Bauer ${ }^{1}$ ) dementsprechend eine starke Steigerung des Sauerstoffverbranches und der Kohlensäureausscheidung, während bei einem Hunde, der durch das Mittel in Narkose verfiel, eine Abnahme des Gasaustausches eintrat. Ebenso fand Rumpf'2) bei Meerschweinchen auf Dosen, welche diese teilweise nicht einmal narkotisierten, einen sehr erheblichen Abfall des Gasumsatzes. Demgegenüber stehen die Versuche von A. Loewy ${ }^{3}$, der beim Menschen im Schlafe nach 0,02 bis 0,025 Morphin einen nur wenig geringeren Verbrauch konstatierte als im natürlichen Schlaf oder bei absoluter Ruhe im wachen Zustande. Diese Unterschiede erklären sich wohl einerseits dadurch, daß die menschlichen Versuchsindividuen sich bei den Normalversuchen riel ruhiger verhielten als die Tiere, grobe Muskelaktion also die als Vergleichshasis dienenden Normalwerte nicht so hoch stellten wie im Tierversuch, andererseits aber besonders durch die ja außerordentlich viel geringeren Dosen, die beim Menschen genügen, um eine Narkose zu erzielen. Sind doch die Dosen, die z. B. Rumpf $f^{4}$ beim Meerschweinchen anwandte, um eine keineswegs sehr tiefe Narkose zu erzielen, auf das Kilo Körpergewicht berechnet fast 1500 mal größer, als die von Loewy bei Menschen applizierten. Es ist also nicht verwunderlich, wenn von diesen hohen Gaben ganz andere Wirkungen ausgingen, als von den kleinen Dosen, die genügten, um die empfindliche, mensehliche Großhirnrinde zu beeinflussen. Ein direkter Einfluß des Morphins anf die Oxydation in den Geweben ist so gat wie ansgeschlossen ${ }^{5}$. Die Wirkung auf den Energieumsatz scheint haupt-

1) a. a. 0 .

2) a. a. 0 .

3) Über den Einfluß einiger Schlafmittel anf die Erregbarkeit des Atemzentrums, nebst einigen Beobachtungen über die Intensität des Gaswechsels im Schlafe des Menschen. Berl. klin. Wochenschr. 1891, 434.

4) a. a. 0 .

5) Man vergleiche damit auch die Ausfuihrungen von Loewi in v. Noordens Handbuch der Pathologie des Stoffwechsels, Bd. II. 
sächlich von dem motorischen Zustande, in den die Tiere durch das Gift versetzt werden, abzuhängen.

In neuerer Zeit ist von seiten einiger unserer besten Forscher ein besonderer Einfluß des Morphins auf die Wärmeregulationszentren betont und mit der Wirkung des Antipyrins verglichen worden $\left.{ }^{1}\right)$. Ich war deshalb darauf gespannt, die Wirkung des Alkaloides anf Tiere ohne zentralen Wärmeregulationsapparat zu beobachten.

Ich fand in 13 Versuchen mit einer einzigen Ausnahme recht erhebliche Herabsetzung der Körpertemperatur.

Das Morphin wurde teils in Form von Pantopon, teils als Morphinum hydrochloricum gegeben und zwar in kleinen Dosen von 0,0027 bis 0,012 pro Kilo Tier.

Das Pantopon wirkte, wie ich durch Einspritzung beider Präparate in entsprechender Dose bei dem gleichen Tiere mehrmals feststellte, quantitativ ziemlich genau seinem (50prozentigen) Morphingehalt entsprechend, so daß ich darauf verzichte, die Versuche mit Pantopon besonders anzuführen. Bei Angabe der Dosis werde ich statt der Menge des Pantopons die halb so große Zahl für Morphin angeben.

Für unsere genauere Betrachtung kommen nur 7 Versuche in Betracht. Die übrigen sind, was die Größe des Ausschlages betrifft, nicht vollwertig; weil keine Temperaturkonstanz voranging 2).

In der Größe des Temperaturabfalles zeigte sich ein bedeutender Unterschied zwischen den nicht gelähmten, am Gehirn operierten Tieren und den Tieren mit durchschnittenem Riuckenmark. Bei letzteren war der Abfall durchweg viel geringer. So fand ich bei einem Tiere mit durchschnittenem Halsmark auf 0,012 Morphin ïberhaupt keinen Ausschlag, der die Fehlergrenze iberschritten hätte $\left(-0,22^{\circ}\right)$ und bei einem weiteren auf 0,0117 einen Abfall am $0,4^{\circ}$, während ein im Gehirn operiertes Tier auf die gleiche Dosis einen Abfall von $2,2^{\circ}$ anfwies. Ein anderes Tier mit Gehirnoperation bekam auf die sebr kleine Dosis von 0,0027 pro Kilo einen Abfall von $0,95^{\circ}$, während zwei weitere Tiere mit durchschnittenem Halsmark (deren Extremitäten übrigens infolge der Unvollständigkeit der Querschnittsläsion bei völlig aufgehobener Wärmeregulation nicht rollkommen gelähmt waren), auf die fast doppelt so große Dosis von 0,005 mit Ausschlägen

1) Gottieb, Arch. f. experiment. Pathol. und Pharmakol. Bd. 26, 1890 und H. Meyer und Gottlieb, Die experiment. Pharmakol., Berlin-Wien 1910; Schmiedeberg, Grundriß der Pharmakol. 7. Auflage, Leipzig 1913.

2) Pantopon gab ich im Anfang meiner Untersuchungen nicht selten zwecks Bekämpfung der Durchtälle, die besonders als Folge der Abkühlung der Tiere, vielleicht auch infolge der unphysiologischen Ernährungsweise häufig auftraten. Später bemerlkte ich, daß mit Tanninpräparaten in dieser Hinsicht mehr zu erreichen ist. 
von - 0,6 und 0,7 reagierten. $O b$ unsere Ausschläge sich allein durch eine Abnahme der Wärmebildung erklären oder ob auch eine Veränderung der Wärmeabgabe eingetreten ist, kann auch hier ohne darauf gerichtete Versuche nicht sicher entschieden werden. Im Gegensatz zu den vielen Untersuchungen, welche einen Einfluß des Vorphins auf die Wärmebildung dartun, habe ich aber in der Literatur keine sicheren Anbaltspunkte für eine Beeinflussung der Wärmeabgabe durch das Gift finden können. Ich nehme also an, daß auch

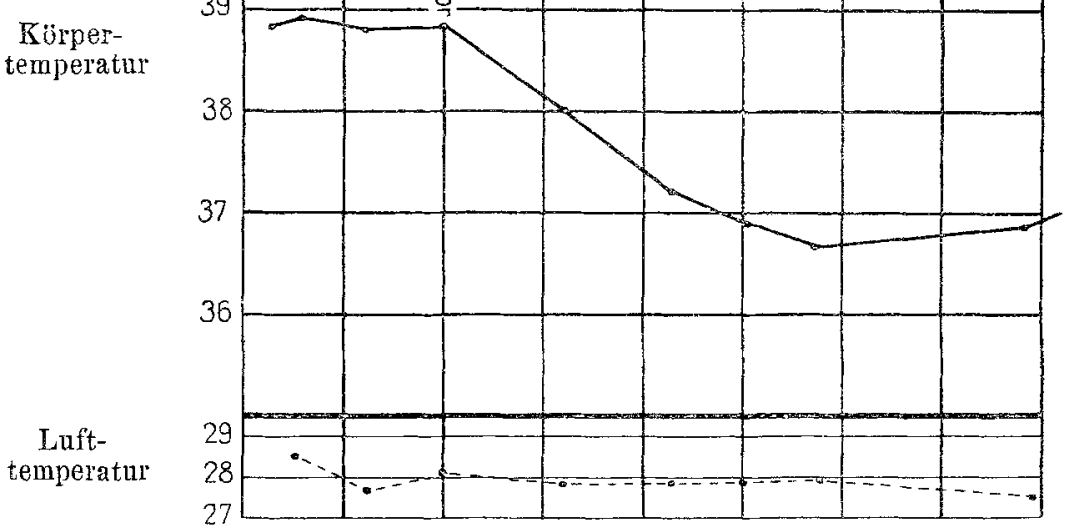

Fig. 4. Wirkung von 0,018 Morphin auf ein Tier mit durchtrenntem Hirnstamm.

hier der Veränderung der Körpertemperatur eine Yeränderung der Wärmebildung entspricht.

Wir schließen aus den Versucben, daß Morphin die Verbrennungen im Organismus auch unabhängig von den zentralen Wärmeregulationsapparaten herabsetzt. Bei gelähmten Tieren ist diese Herabsetzung sehr gering. Sie läßt sich also durch den Einfluß auf die Muskelinnervation, speziell auch die Atemmuskulatur - wie übrigens seit langem von vielen Seiten angenommen wurde - erklären.

Wir haben noch die Frage zu erörtern, ob und inwieweit die Annahme von einer besonderen Einwirkang des Morphins anf die Wärmeregulationszentren durch unsere Befunde beribht wird. Die Annahme stützt sich vor allem auf Versuche vou Gottlieb ${ }^{1}$ ). Kaninchen von 1,75 bis $1,9 \mathrm{~kg}$

1) Areh. f. experiment. Pathol. und Pharmokol. Bd. 26, 1890. 
Gewicht bekamen während der Temperatursteigerung nach dem Wärmestich 10 bis $20 \mathrm{mg}$ Morphin und reagierten darauf mit einem vorübergehenden Temperaturabfall von 1,0 bis $1,5^{\circ}$. Von dem Autor und anch von Schmiedeberg ${ }^{1}$ ) wird betont, daß solche Dosen die Kaninchen nicht narkotisieren. Da andererseits ein direkter Einfluß des Morphins auf die Stätten des Stoffwechsels auszuschließen ist, liegt die Annahme einer Lähmung and Beruhigung der durch den Gehirnstich gereizten Nervengebiete, wie sie etwa das Antipyrin bewirkt, sehr nahe. Bei dem starken Einfaß, den das Morphin auf die Körpertemperatur meiner Tiere nach der Ausschaltung der der Wärmeregulation vorstehenden Hirnteile in ebenso kleinen und noch kleineren Dosen ausübte, ist es mir zweifelhaft geworden, ob die Wirkung anf die Temperatur der Kaninchen nach dem Wärmestich wirklich mit Recht mit derjenigen des Antipyrins verglichen wird. Ist doch die Größe des Temperaturabfalles, der in den erwähnten Versuchen während der Wärmestichhyperthermie erzielt wurde, sogar etwas kleiner als derjenige, den ich regelmäßig bei meinen Tieren ohne Wärmeregulationszentrum beobachtete.

Kaninchen 53 z. B. hatte, wie schon erwähnt, auf eine Dosis von 11 bis $12 \mathrm{mg}$ pro Kilo, entsprechend also den größeren von Gottlieb angewandten Dosen, einen Abfall von 2,2 $2^{\circ}$ das Tier 27 anf nur $2,7 \mathrm{mg}$, auf eine Dosis also, die nur halb so groß ist, wie die kleinste von Gottlieb in seinen Wärmestichversuchen applizierte, einen Abfall von $0,95^{\circ}$.

Es ist natürlich auch Gottlieb nicht entgangen, daß die von ihm gegebenen kleinen Morphindosen das ganze Tier deutlich beeinflussen und er hat die Möglichkeit einer Beeinflussung der Wärmebildung auch in Betracht gezogen. Ich möchte aber mehr Gewicht darauf legen, wie stark der Einfluß dieser Dosen von 0,01 bis 0,02 regelmäßig auch auf andere Funktionen des Nervensystems unserer Versuchstiere ist. Selbst auf die um ein mehrfaches niedrigere Dosis von einem Milligramm pro Kilo Tier hat Impens 2) eine sehr deutliche Abnahme der Frequenz und des Volumens der Atmung nachgewiesen, und ich kann bestätigen, daß auf Dosen ron 2 bis $3 \mathrm{mg}$ pro Kilo sowohl bei operierten als bei normalen Tieren eine Verlangsamung der Atmung so gat wie ausnahmslos besteht. Der Muskeltonus ist schon bei so kleinen Dosen fast immer, bei etwas größeren, wie sie Gottlieb angewandt hat, aber ausnahmslos am ganzen Körper dentlich herabgesetzt. Die Tiere sträubten sich gegen die Temperaturmessung fast immer erheblich weniger, manche sind deutlich schläfrig. Es besteht also jedenfalls anch bei diesen kleinen Dosen die Möglichkeit, daß tiefer liegende und nicht durch den Wärmestich unmittelbar erregte Nervengebiete in ibrer Funktion so verändert werden, daß sie den Wärmehaushalt beeinflussen. Es scheint mir also, daß, solange kalorimetrische Versuche die Frage, woranf die Wirkung des Morphins auf die Wärmestichhyperthermie des Kaninchens beruht, nicht endgültig entschieden haben, die Annabme einer durch Vermittlnng niedrigerer Nervenzentren bewirkten Abnahme der Wärmebildung mebr Wahrscheinlichkeit für sich hat, als ein der Antipyrinwirkung entsprechender Vorgang.

1) a. a. O. S. 267.

2) Über die Wirkung des Morphins und seiner Derivate auf die Atmnng. Pflügers Arch. Bd. 78, 1899. 
Daß in hohen Dosen von 30 bis $50 \mathrm{mg}$ (bei Tieren von etwa $2 \mathrm{~kg}$ Gewicht) die zentrale Wärmeregulation durch Morphin gestört werden kann, ist durch den Nachweis, daB so vergiftete Tiere sich bei hohen Lufttemperaturen leichter überbitzen als normale, dureh Gottlieb ${ }^{1}$ ) mittels einer sehr sinnreichen Versuchsanordnung einwandfrei festgestellt. Nicht narkotische Dosen hatten aber eine solehe Wirkung nicht, so daß eine besonders große Empfindlichkeit des zentralen Wärmeregulationsapparates gegen das Morphin ans diesen-Versuchen nicht herrorgeht.

\section{Zusammenfassung.}

Bei Kaninchen, die nach Ausschaltung der zentralen Wärmeregulation in gleichmäBiger Temperatur gehalten werden, sind die auf toxische Einwirkungen stattfindenden Temperatnrschwankungen eiu direkter Ausdruck der Schwankungen der Wärmebildung, respektive des Energieumsatzes.

Natrium salicylicum ruft auch in kleineren Gaben regelmäßig eine erbebliche Steigerung der Wärmebildung hervor und zwar unabhängig von einer etwa auftretenden motorischen Erregung.

Antipyrin bringt bei gelähmten Tieren keine Veränderung des Energieumsatzes hervor, bei nicht gelähmten dagegen meistens eine geringe Steigerung, welche durch die motorische Unruhe der Tiere gentigend erklärt wird.

Auf Chinin in mittleren Dosen findet in allen Versuchen eine Abnahme der Wärmebildung statt. Bei höheren Dosen wird diese Abnahme oft durch die Folgen der motorischen Erregung auf den Stoffumsatz rerdeckt.

Morphin setzt schon in kleinsten Dosen die Wärmebildung herab. Da diese Wirkung bei gelähmten Tieren viel geringer ausfällt, ist sie wahrscheinlich eine Folge der Herabsetzung der Motilität.

1) a. a. 0 . 